\title{
Preparation of Modified Hollow Polypropylene Membrane and Their Adsorption Properties of $\boldsymbol{\gamma}$-Globulins
}

\author{
Taek-Sung Hwang* and Jin-Won Park \\ Department of Chemical Engineering, Chungnam National University, Gung Dong, Yuseong, Daejeon 305-764, Korea
}

Received May 13, 2003; Revised July 16, 2003

\begin{abstract}
The hydrophobic ligand-containing hollow polypropylene (PP) membranes were synthesized by the mutual radiation induced graft copolymerization with glycidylmethacrylate (GMA) onto hollow PP membrane followed by the subsequent functionalization with L-phenylalanine. FT-IR, elemental analysis and UV spectroscopy were utilized to characterize copolymer composition, and degree of grafting, functionalization conversion and $\gamma$-globulins adsorption. The degree of grafting on the PP surface increased with the reaction time and total dose of E-beam. In the subsquent functionalization, the amount of L-phenylalanine increased with the increase in the degree of grafting and the degree of conversion was about $30 \%$. The $\gamma$-globulins adsorption experiments showed that adsorption capacity had a maximum value at $\mathrm{pH} 8$. The $\gamma$-globulins adsorption capacity in the basic $\mathrm{pH}$ region was higher than in the acidic $\mathrm{pH}$ region.
\end{abstract}

Keywords: hydrophobic interaction, hollow membrane, adsorption, $\gamma$-globulins, L-phenylalanine.

\section{Introduction}

Within a context of rapidly increasing demand for effective separation and purification in biotechnology, pharmacy, and chemical/wastewater treatment, studies of separation using membranes are being watched with keen interest. ${ }^{14}$ The membrane for the separation and purification of protein has been divided into two classes according to the membrane shape: flat sheet membranes and hollow membranes. The hollow membranes have been more effective than the flat sheet membranes, because the hollow membranes have more specific mean pore diameters for the separation of proteins, and can more easily facilitate the organization of a compact module. $^{5}$

Recently, commercial hollow membranes used the separation of protein, called hydrophilic membranes, have been used conventionally for size-based separation processes. But these membrane have a disadvantage that their pores get filled during the separation process. More recently, membranes containing functional groups were investigated to improve the disadvantages of size-based separation. In a study on that exact issue, protein was desorbed in membrane by various conditions such as $\mathrm{pH}$, after ion exchange or hydrophobic ligand functional group-containing membranes were adsorbed the protein. ${ }^{6-9}$

The membranes containing functional groups are used

*e-mail : tshwang@ cuvic.cnu.ac.kr

1598-5032/10/347-05 @ 2003 Polymer Society of Korea more for the separation or purification of protein than for the medical uses. The membranes containing functional groups have been divided into three classes: ion exchange membranes (IEX), affinity membranes, and hydrophobic interaction membranes (HI), according to the chemical interactions between the functional group and protein. ${ }^{10} \mathrm{HI}$ membranes are especially used in combinations by van der Waals interaction between a hydrophobic ligand on the surface and a protein. Functional groups that have protein and hydrophobic interaction are phenyl, octyl, polyethylene glycol, and L-phenylalanine. However, HI membranes which have studied recently, have been shown that the functional groups crosslink directly to membranes. It is difficult to introduce the functional groups to membrane pore, so the degree of the purity in the separated and purified protein is low. ${ }^{11-15}$

Therefore in this study, to introduce functional group to whole membrane, the hydrophobic ligand-containing hollow PP membranes were synthesized by the mutal radiation induced graft copolymerization with GMA onto hollow PP membrane followed by the subsequent functionalization with L-phenylalanine. We confirmed a change of mean pore diameter according to the degree of grafting and functionalization condition, and after we investigated $\gamma$-globulins adsorption properties by $\mathrm{pH}$ and adsorption time using $\mathrm{HI}$ hollow PP membrane which were synthesized, examined optimum adsorption condition. 


\section{Experimental}

Materials. Hollow PP membranes used as trunk polymers, with a mean pore diameter of $2 \mu \mathrm{m}$ and $70 \%$ porosity, were used courtesy of a purchase of Accurel $^{\circledR}$ hollow PP membranes from Membrana Co. Germany. GMA was purchased from Junsei Chemical Co. Japan and was used after removal of hydroquinone. L-phenylalanine that was used by the functionalization reagent and $\gamma$-globulins were from the Aldrich Chemical Co. USA, and Sigma Chemical Co. USA, respectively. Other solvents were used after being obtained from Duksan Chemical Co., Korea. All reagents and chemicals used in these studies were of analytical grade.

Grafting Copolymerization of GMA. GMA grafted hollow PP copolymers were synthesized by E-beam mutal radiation induced graft copolymerization with GMA onto hollow PP membrane according to path of Figure 1. Hollow PP membrane and GMA was irradiated in nitrogen atmosphere with 15, 20 and $20 \mathrm{Mrad}$ of $2 \mathrm{MeV}$ E-beam (ELV-4,
EB tech Co., Daejeon, Korea).

To synthesize GMA-grafted hollow PP copolymers, hollow PP membrane was washed with acetone and distilled water and then was dried in a $60^{\circ} \mathrm{C}$ dry oven. Half a gram of dried hollow PP membranes was placed with monomers of several concentrations into an inner diameter $4 \mathrm{~mm}$ reactor, and was then substituted by nitrogen for $2 \mathrm{hrs}$ and swelled over the course of $12 \mathrm{hrs}$. Table I shows experiment conditions involving monomer concentrations and total dose amounts.

After the grafting reaction, and until we perfectly removed the homopolymer and unreacted monomer by benzene with no subsequent weight decrease, we vacuum dried copolymers in $60{ }^{\circ} \mathrm{C}$ lower, and we then calculated the degree of grafting according to the equation (1).

$$
\text { Degree of grafting }(\%)=\frac{W_{g}-W_{0}}{W_{o}} \times 100
$$

where $W_{g}$ is the weight of grafting copolymer, $W_{0}$ is the

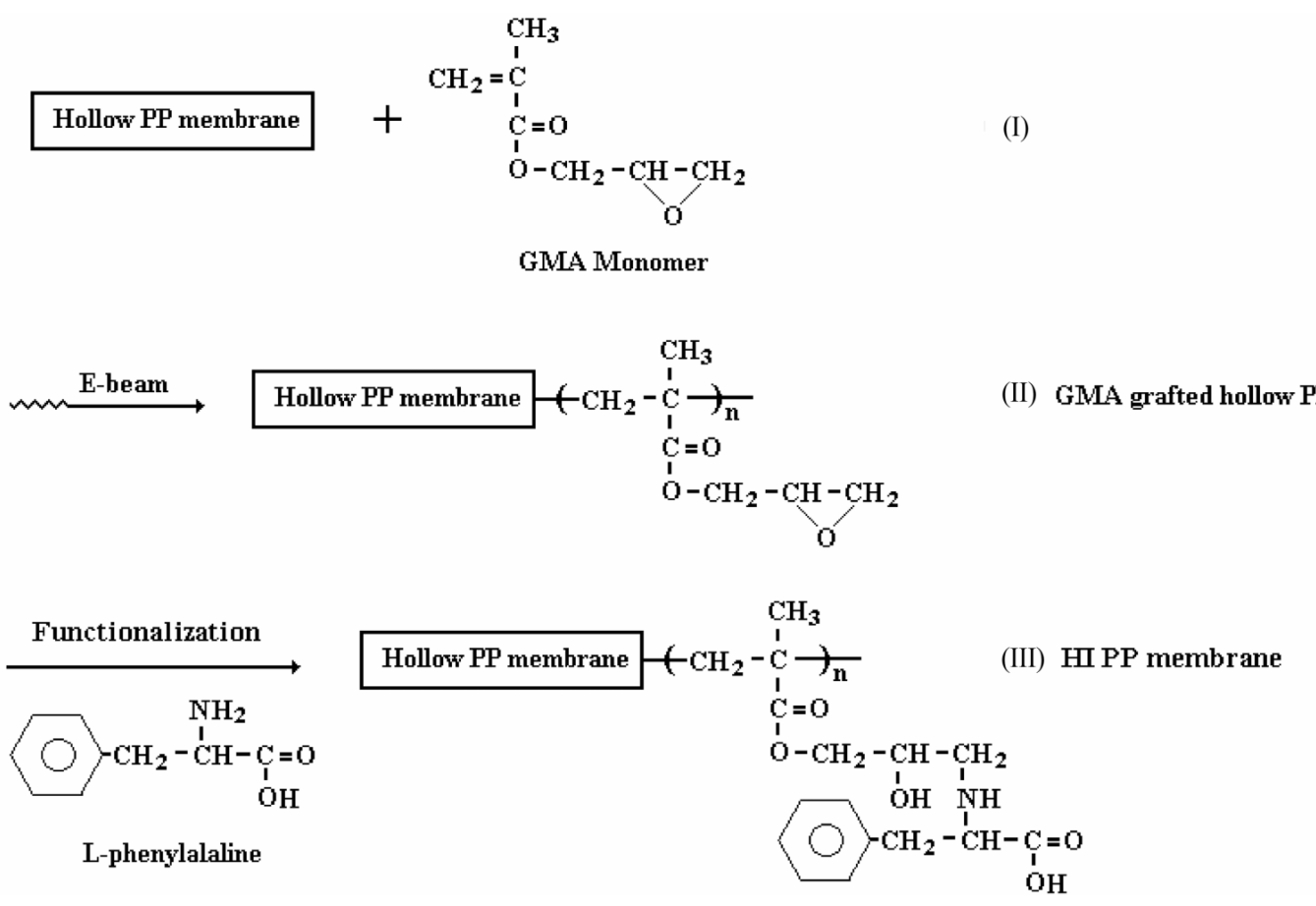

Figure 1. Synthetic mechanism of HI hollow PP membrane by electron beam accelerator.

Table I. Synthetic Conditions of Hollow GMA-g-PP Copolymers

\begin{tabular}{cccccccc}
\hline Exp. No. & Hollow PP $(\mathrm{g})$ & GMA $(\mathrm{vol} \%)$ & Methanol $(\mathrm{vol} \%)$ & \multicolumn{2}{c}{ Total Dose $(\mathrm{Mrad})$} & Reaction Temp. $\left({ }^{\circ} \mathrm{C}\right)$ \\
\hline 1 & 0.5 & 20 & 80 & 15 & 20 & 25 & 50 \\
2 & 0.5 & 40 & 60 & 15 & 20 & 25 & 50 \\
3 & 0.5 & 60 & 40 & 15 & 20 & 25 & 50 \\
4 & 0.5 & 80 & 20 & 15 & 20 & 25 & 50 \\
5 & 0.5 & 100 & 0 & 15 & 20 & 25 & 50 \\
\hline
\end{tabular}


weight of PP trunk polymer used.

Functionalization Reaction. ${ }^{16}$ To introduce the L-phenylalanine functional group that has a selective adsorption property about $\gamma$-globulins to GMA-grafted hollow PP copolymer, we put copolymers in a solution which added copolymer to $\mathrm{K}_{2} \mathrm{CO}_{3} 100 \mathrm{~mL}$ with $5 \mathrm{~g}$ of L-phenylalanine, $0.2 \mathrm{~g} \mathrm{NaNO}_{2}$, and then functionalized over $24 \mathrm{hrs}$. After the functionalization reaction, washed $\mathrm{HI}$ hollow PP membrane, which were functionalized by acetone and distilled water, were then dried in a $60^{\circ} \mathrm{C}$ dry oven. We then calculated the amount of the L-phenylalanine group according to the elemental analysis (EA 1110, CE Instruments, Milan, Italy).

Analysis of HI Hollow PP Membrane. The Fourier transform-infrared (FT-IR) spectra were obtained on an FTIR spectrometer (Galaxy 5020, Mattson Instruments, Madison, USA) over the region $4000 \sim 400 \mathrm{~cm}^{-1}$, resolution $4 \mathrm{~cm}^{-1}$ to confirm the structure of HI hollow PP membrane, which was synthesized through a grafting reaction and functionalization. The contents of $\mathrm{C}, \mathrm{H}$ and $\mathrm{N}$ that were introduced onto HI hollow PP membrane were analyzed by a elemental analyzer. The mean pore diameter changes that occurred as a result of the grafting copolymerization and functionalization were analyzed for confirmation by nitrogen adsorption methods employed by using a BET analyzer (TriStar 3000, Micromeritics, Norcross, USA).

$\boldsymbol{\gamma}$-Globulins' Adsorption Properties of HI Hollow PP Membrane. $\gamma$-globulins' adsorption properties of HI Hollow PP membrane were measured in an adsorption experiment that took place after regulating the $\mathrm{pH}$ range from 4 to 10 with a phosphate and $\mathrm{NaOH}$ buffer solution. The initial concentration of $\gamma$-globulins and its adsorption temperature were fixed at $200 \mathrm{ppm}$ and $25^{\circ} \mathrm{C}$ respectively. The effluent was continuously sampled at scheduled time, and the amount of the $\gamma$-globulins adsorption was calculated by UV adsorption spectroscopy (UV-3101, Shitmadzu, Kyoto, Japan).

\section{Results and Discussion}

The degree of grafting and changes of mean pore of $\mathrm{HI}$ hollow PP membrane were shown in Figure 2 as a function of reaction time. As shown in Figure 2, the degree of grafting of the copolymer, according to the reaction time, increased rapidly until $20 \mathrm{~min}$ of reaction time, and after this the increase in width was mitigated. In this way, that degree of grafting, which increased rapidly until $20 \mathrm{~min}$ of reaction time, was considered to be "fast" by grafting reaction standards, because the reaction velocity with radical and monomers which were early created by E-beam radiation at reaction time, was also fast, as was the mitigation of the increase in width. It was considered by the grafting reaction to have happened early in the reaction. On the other hand, the mean pore diameter of membranes decreased rapidly until 20 min with the reaction time, and was reached in equilibrium. In the early reaction time, the pore was broken and narrowed by grafting reaction. As result, the mean pore diameter decreased sharply.

Figure 3 showed the degree of grafting of hollow PP membrane as a function of the total dose. As shown in Figure 3, while the total dose was increased, the degree of grafting increased consistently and rapidly until 10 min reaction time regardless of the total dose. This happened because free radicals which E-beam radiations created on trunk polymers, were increased as the total amount of radiation energy. This result was well fit to the results of Hosfall et al. ${ }^{17}$

Figure 4 showed the degree of grafting of copolymers which were synthesized by changing monomer concentration in $25 \mathrm{Mrad}$ of total dose during $90 \mathrm{~min}$ of reaction time. As shown in Figure 4, the GMA monomer concentration was increased, the degree of grafting was increased, and the maximum degree of grafting was $310 \%$ in an 80 vol\% GMA concentration. The reason is because the monomer concentration increased under solvent existence, and the degree of

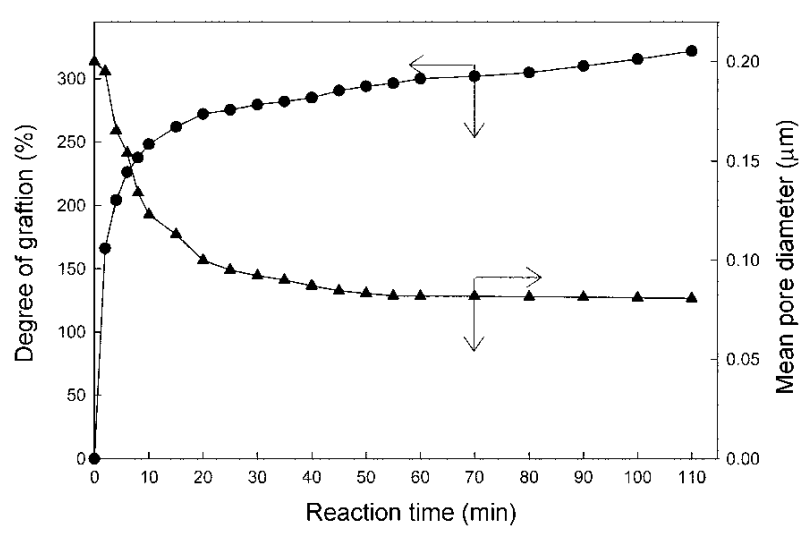

Figure 2. Effect of radiation time on the degree of grafting and mean pore diameter of the hollow PP membrane (total dose : 25 Mrad, and 80\% GMA / $20 \%$ methanol).

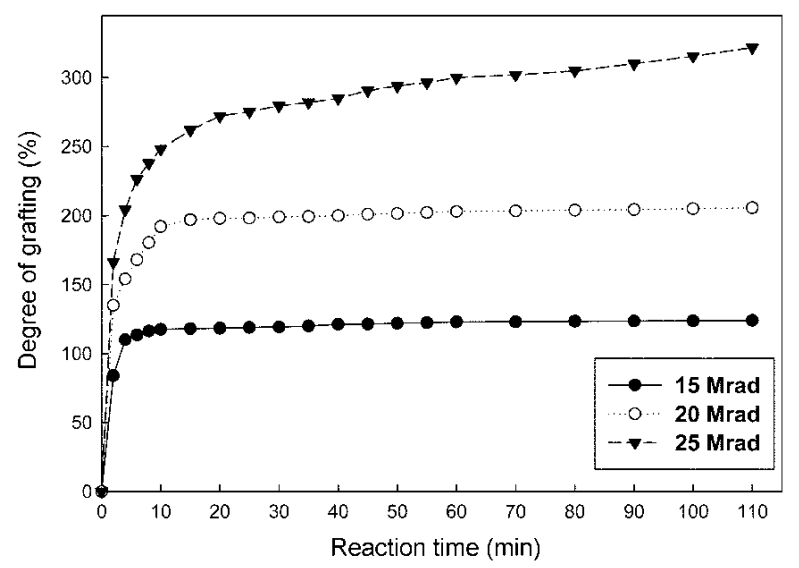

Figure 3. Effect of total dose on the degree of grafting onto the hollow PP membrane (80\% GMA / 20\% methanol). 
grafting was increased to some extent when enough swelling of methanol, a solvent activated by E-beam radiation, occurred in a trunk polymer. Also, the swelling of trunk polymers progressed to membrane's pore interior by contact with monomer.

On the other side, in case solvents did not exist, the amount of homopolymer was increased due to occurring the grafting reaction on the surface of membrane, we considered the obtained degree of grafting to be "low".

Figure 5 showed ${ }^{18}$ the FT-IR spectra of copolymers which were synthesized by grafting reaction and functionalization. As shown in Figure 5(b), it was observed that carbonyl ( $>\mathrm{C}=\mathrm{O}$ ) and epoxy (-COC-) stretching vibration peaks, which were characteristic peaks of GMA by grafting reaction, appear 1720 and $910 \mathrm{~cm}^{-1}$ and could confirm that grafting copolymer was synthesized. Figure 5(c) spectra was FT-IR spectra for functionalization confirmation. The characteristic

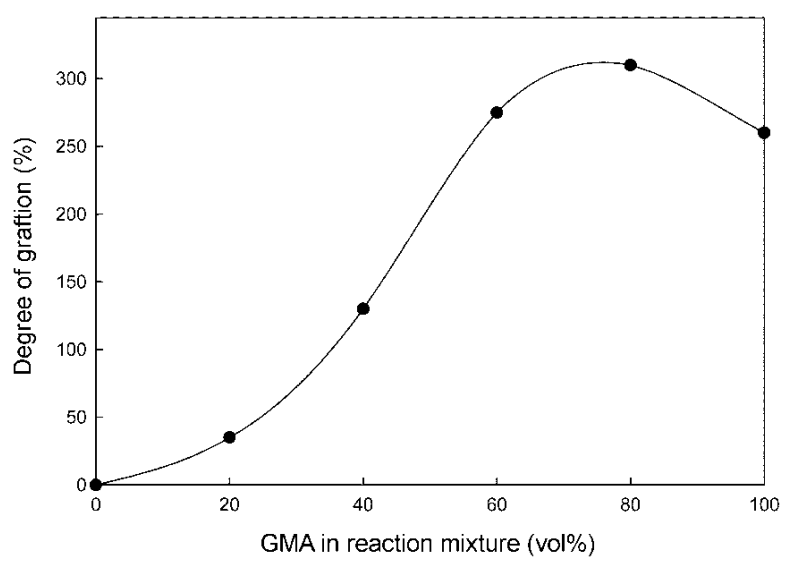

Figure 4. Effect of monomer concentration on the degree of grafting onto the hollow PP membrane (reaction time : $90 \mathrm{~min}$, and total dose : $25 \mathrm{Mrad}$ ).

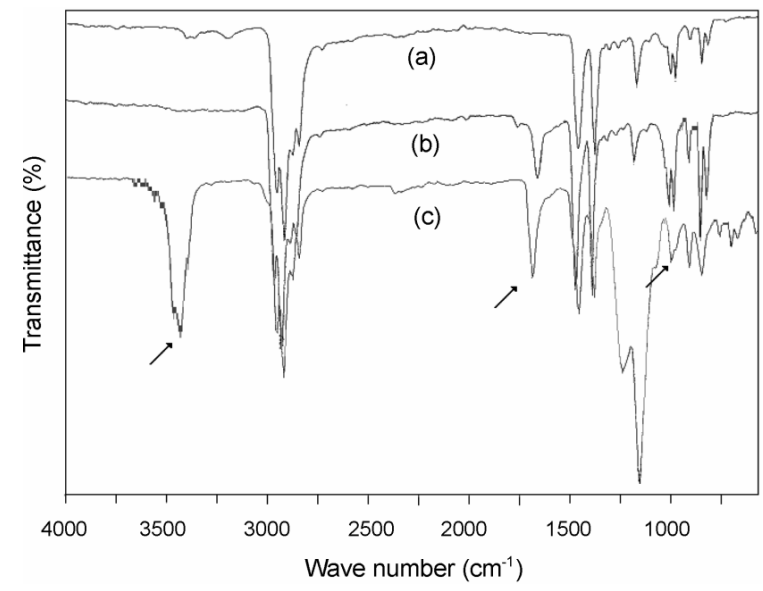

Figure 5. FT-IR spectra of hollow PP membranes (a) hollow PP membrane, (b) hollow GMA-g-PP, and (c) HI hollow PP membrane. peak intensity of epoxy at $910 \mathrm{~cm}^{-1}$ decreased remarkably, and the new broad absorption peaks came at $3500 \mathrm{~cm}^{-1}$ due to secondary amine (-NH) and hydroxyl $(-\mathrm{OH})$ that appeared in Figure 5(c). Besides, we confirmed that an L-phenylalanine group was introduced to copolymer observing a stretching vibration peak of aromatic - $\mathrm{CH}$ - at $3030 \mathrm{~cm}^{-1}$ and characteristic peak of benzene at $1450 \sim 1600 \mathrm{~cm}^{-1}$.

Figure 6 showed the elemental analysis performed to confirm the amount of L-phenylalanine, which was introduced according to degree of grafting. As shown in Figure 6, the amount of L-phenylalanine which was introduced increased rapidly at $248 \%$ of degree of grafting and reached equilibrium; conversion was about 30 percent.

The $\gamma$-globulins adsorption properties of HI hollow PP membrane, as a function of adsorption time at $\mathrm{pH} 7$ and $25^{\circ} \mathrm{C}$ of adsorption temperature, were shown in Figure 7. The $\gamma$-globulins adsorption rate until an adsorption time of

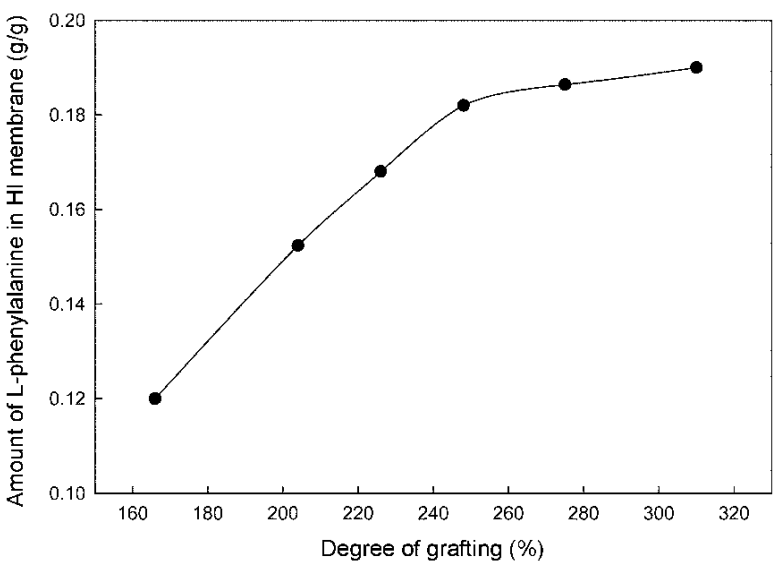

Figure 6. Plot of amount of L-phenylalanine in HI membrane and degree of grafting onto the hollow PP membrane.

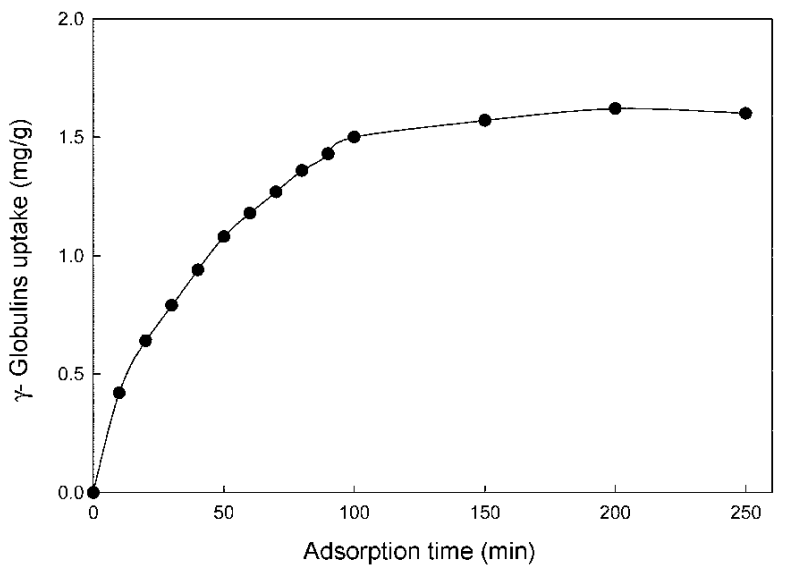

Figure 7. Plot of content and adsorption time of $\gamma$-globulins on the HI hollow membrane (Initial concentration of $\gamma$-globulins $200 \mathrm{ppm}, \mathrm{pH} 7$, and adsorption temperature $25^{\circ} \mathrm{C}$ ). 


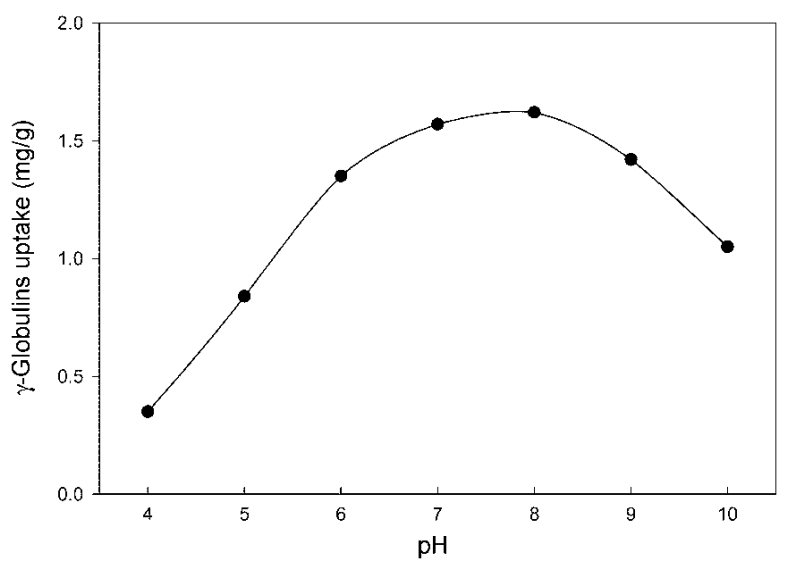

Figure 8. Effect of $\mathrm{pH}$ on the $\gamma$-globulins adsorption of HI hollow membrane (Initial concentration of $\gamma$-globulins $200 \mathrm{ppm}$, adsorption time $150 \mathrm{~min}$, and adsorption temperature $25^{\circ} \mathrm{C}$ ).

$50 \mathrm{~min}$ was very fast, and reached equilibrium at $100 \mathrm{~min}$. Like Kubota, et al. ${ }^{11}$ which studied in an adsorption experiment of BSA using HI hollow PP membrane which contained phenyl, this result showed same result that the $\gamma$ globulins had a fast material transfer into the HI hollow PP membrane interior.

Figure 8 showed the $\gamma$-globulins adsorption performance according to the various $\mathrm{pH}$ levels. The maximum amount of adsorption of $\gamma$-globulins appeared in $\mathrm{pH} 8$, and the adsorption amount in the basic region appeared higher than that in the acidic region.

\section{Conclusions}

The HI hollow PP membrane was synthesized by the mutual radiation-induced graft copolymerization with GMA onto hollow PP membrane, followed by the subsequent functionalization with L-phenylalanine. The degree of grafting of copolymer by reaction time increased rapidly until 20 min, and the mean pore diameter of membrane decreased rapidly until $20 \mathrm{~min}$ according to the reaction time. The degree of grafting of hollow PP membrane increased along with increases in the total dose, and it increased rapidly until 10 min without relation to the total dose. As the GMA concentration was increased, the degree of grafting increased and obtained $310 \%$ of the maximum degree of grafting in an $80 \mathrm{vol} \%$ of GMA concentration. The amount of L-phenylalanine was the highest in $248 \%$ of the degree of grafting by functionalization, and the conversion of L-phenylalanine was about $30 \%$. The result of the $\gamma$-globulins adsorption experiment of HI hollow PP membrane was that the adsorption rate was very fast until $50 \mathrm{~min}$, and afterwards reached equilibrium in $100 \mathrm{~min}$. The maximum adsorption capacity according to the various $\mathrm{pH}$ levels was obtained at $\mathrm{pH} 8$; the amount of $\gamma$-globulins adsorption in the basic region appeared higher than in the acidic region.

\section{References}

(1) G. H. Yang, E. T. Kang, and K. G. Neoh, Appl. Surf. Sci., 178, 165 (2001).

(2) H. A. A. El-Rehim, E. A. Hegazy, and A. E. Ali, React. Funct. Polym., 43, 105 (2000).

(3) R. Ghosh, S. S. Silvia, and Z. Cui, Biochem. Eng. J., 6, 19 (2000).

(4) I. Noh and J. H. Lee, Korea Polym. J., 9, 197 (2001).

(5) S. S. Kim and J. J Kim, Polymer Sci. Tech., 7, 148 (1996).

(6) D. Okamura, K. Saito, K. Sugita, M. Tamada, and T. Sugo, J. Chromatogr. A, 953, 101 (2002).

(7) G. Bayramoglu, M. Yilmaz, and M. Y. Arica, Biochem. Eng. J., 3651, 1 (2002).

(8) K. Kato, E. Uchida, E. T. Kang, Y. Uyama, and Y. Ikada, Prog. Polym. Sci., 28, 209 (2002).

(9) H. Zou, Q. Luo, and D. Zhou, J. Biochem. Biophys. Methods, 49, 199 (2001).

(10) R. Ghosh, J. Chromatogr. A, 952, 13 (2002).

(11) N. Kubota, M. Kounosu, K. Saito, K. Sugita, K. Watanabe, and T. Sugo, J. Chromatogr. A, 718, 27 (1995).

(12) T. B. Tennikova and F. Svec, J. Chromatogr. A, 646, 279 (1993).

(13) J. A. Queiraz, F. A. P. Garcia, and J. M. C. Cabral, J. Chromatogr. A, 734, 213 (1996).

(14) M. Y. Arica, G. Akin-Oktem, and A. Denizli, Colloids Surf., B : Biointerfaces, 21, 273 (2001).

(15) T. S. Hwang and J. H. Lee, Polymer(Korea), 26, 160 (2002).

(16) M. Kim, K. Saito, S. Furusaki, T. Sugo, and I. Ishigaki, $J$. Chromatogr. A, 586, 27 (1991).

(17) J. A. Horsfall and K. V. Lovell, Eur. Polym. J., 38, 1671 (2002).

(18) S. H. Choi, Y. M. Hwang, and K. P. Lee, J. Chromatogr. A, 987, 323 (2003). 